\title{
DESMINADO HUMANITARIO: IMPACTO SOCIOECONÓMICO EN EL MUNICIPIO DE SAN VICENTE DE CHUCURÍ, SANTANDER, COLOMBIA*
}

\author{
Humanitarian demining: socio-economic impact in the \\ municipality of San Vicente de Chucurí, Santander, Colombia
}

\author{
Elcida Muñoz Villamizar \\ Magíster en Fiscalidad Internacional, Universidad Internacional La Rioja. \\ Grupo de Investigación VIDOCQ, Universidad de Investigación y \\ Desarrollo - UDI (Bucaramanga, Colombia).
} Correo electrónico: emunoz6@udi.edu.co

| Recibido/Received: 13/01/2021 • Aprobado/Approved: 29/06/2021 • Publicado/Published: 30/07/2021

Resumen

El proceso de desminado humanitario se ha concebido en un contexto de descontaminación del territorio y de atención integral a las víctimas de las minas antipersonales ,entendidas estas como un artefacto empleado en el conflicto armado, cuyo uso ha derivado en graves consecuencias para la comunidad. Por lo anterior, en el presente artículo se analiza el proceso del desminado humanitario en el municipio de San Vicente de Chucurí, siendo este uno de los primeros municipios de Santander, en ser declarado como libre de sospecha de minas. A partir del análisis descriptivo-exploratorio, que se implementó para esta investigación, se buscó determinar el impacto del desminado humanitario en el municipio antes señalado. Se concluye aquí en la necesidad de su implementación, como una garantía para el efectivo uso de la tierra y de las labores del campo, sin los riesgos que puedan devenir de la acción de las minas antipersonales; así mismo, dicho desminado se considera como uno de los componentes necesarios, en alineación con estrategias políticoadministrativas, para lograr la consolidación de la reconstrucción social.

Palabras clave: conflicto armado, derechos humanos, desarrollo socio económico, minas antipersonal, seguridad.
Abstract
The humanitarian demining process has been conceived in a context of decontamination of the territory and integral attention to the victims of antipersonnel mines, as an artifact used in the armed conflict whose use has had serious consequences for the community. Therefore, this article analyzes the process of humanitarian demining in the municipality of San Vicente de Chucurí, one of the first municipalities in Santander to be

\footnotetext{
*Artículo de tipo no experimental, de enfoque cualitativo, resultado de un proyecto de investigación culminado, perteneciente al área de Ciencias Sociales y Humanidades, subárea de Posconflicto, el cual fue desarrollado en el Grupo de Investigación VIDOCQ. Este fue financiado por la Universidad de Investigación y Desarrollo -UDI- (Bucaramanga, Colombia).
} 
declared free of suspected mines. The descriptive-exploratory analysis seeks to determine the impact of humanitarian demining in the municipality of San Vicente de Chucurí; it concludes on the need for its implementation as a guarantor of the use of land and field work without risks due to the action of antipersonnel mines, as well as one of the necessary components in alignment with political-administrative strategies aimed at the consolidation of social reconstruction.

Key words: Armed conflicts, human rights, socio-economic development, antipersonnel mines, security.

\section{INTRODUCCIÓN}

Existen diferentes factores que influyen en el nivel de crecimiento y en el desarrollo de una localidad. Su análisis se deriva de los aspectos económicos, políticos y de seguridad, los cuales se componen de varios elementos complementarios y en su articulación permiten la solución de necesidades básicas como la salud, la educación y la vivienda, entre otras. Es así como las decisiones, estructuras y características de cada sector, determinan las oportunidades para el desarrollo económico y, con ello, se generan condiciones de convivencia y de seguridad.

La articulación de las políticas nacionales, regionales y locales es considerada como una herramienta de crecimiento, tal como también lo es la participación de los ciudadanos; igualmente, se considera relevante tener conocimiento sobre las diferentes problemáticas, todo lo anterior, con el objetivo de lograr la construcción de soluciones acertadas.

Las características que se tienen respecto del municipio de San Vicente de Chucuri, muestran una alternancia entre las diferentes etapas de la violencia, las cuales se observan a través de aspectos como el nacimiento de los grupos guerrilleros, las diferencias entre e los grupos políticos, el surgimiento de las autodefensas, además del enfrentamiento entre los grupos armados ilegales, los cuales buscaban tanto el control del territorio, como el control de las vías de acceso y la movilización de sus integrantes y cabecillas.

San Vicente de Chucurí ha basado su economía principalmente en la producción del cacao, seguido por el cultivo del café, constituyéndose dichas actividades en el sustento económico familiar en la región; por lo tanto, todo cambio en la producción y en la comercialización afecta directamente la capacidad adquisitiva básica y la calidad de vida de sus habitantes.

Dada su ubicación cercana al puerto petrolero de Barrancabermeja, la región presentó los mismos niveles de violencia y la injerencia de todos los actores de la violencia, los cuales se establecieron en el sector, generándose tras ello, un alto índice de hechos delictivos y de inseguridad, lo que a su vez provocó enfrentamientos y la disposición de minas antipersonales; a raíz de estos hechos se presentó un alto número de víctimas y tras ello, también el fenómeno del desplazamiento forzado, así como la llegada de una población flotante, convirtiéndose la región en un sector receptor de los desplazados de otras regiones, los cuales conformaron invasiones, sectores sin recursos económicos, con ausencia de los servicios públicos básicos y un alto grado de hacinamiento. No obstante, dadas estas problemáticas, la región mencionada se convirtió en el primer municipio del departamento de Santander, en el cual se desarrolló el desminado humanitario; por su parte, el panorama señalado motivó la investigación que derivó en el desarrollo del presente artículo. 


\section{METODOLOGÍA}

\section{Tipo de estudio}

El presente estudio es (i) de enfoque cualitativo; (ii) de alcance descriptivo- exploratorio; (iii) con un diseño no experimental de la teoría y con un análisis de datos referido al antes y al después del proceso de desminado humanitario.

\section{Participantes}

El presente artículo tiene como sustento el análisis del impacto del proceso de desminado humanitario, las políticas administrativas y la estadística delictiva, en el municipio de San Vicente de Chucurí, en el departamento de Santander.

\section{Materiales e instrumentos}

Desde la construcción de un marco teórico sobre el empleo de las minas antipersonales, en el desarrollo del conflicto armado; el proceso de desminado humanitario para su destrucción; el posconflicto; la unidad de Restitución de Tierras y el análisis de los aspectos socio económicos y de seguridad del municipio de San Vicente de Chucurí.

\section{Procedimiento}

La investigación se desarrolla a partir de los siguientes ítems. (a). Se organiza un marco teórico, correspondiente a los antecedentes de la violencia generada por el conflicto armado y al uso de las minas antipersonal. (b) Se efectúa un análisis del proceso del desminado humanitario, su metodología, su procedimiento y sus alcances. (c) Se realiza una recopilación de datos, correspondiente a las políticas públicas alternas, implementadas para la atención de las víctimas. (d) Se presenta un análisis del municipio de San Vicente de Chucurí, relacionándolo con su historia y con los planes de desarrollo 2016-2019-2020-2023, generando además un comparativo de los índices delictivos del antes, durante y después de realizado el proceso de desminado humanitario en la localidad. (e) Se concluye con la formulación de la idea de que es necesaria la implementación de estrategias de reconstrucción social, una vez realizado el proceso de desminado humanitario.

\section{Conflicto armado}

Como fundamentación teórica se requiere conocer primero las características del conflicto armado, dada su vinculación directa con el uso de las minas antipersonales. Por tal motivo, se realizó un análisis de sus características, las cuales han sido objeto de estudio, desde diferentes ángulos, partiendo del estudio de su origen, duración, actores, artefactos empleados, efectos sobre las victimas y las poblaciones donde se desarrolló. En relación al origen, este se ha se sustentado desde las "confrontaciones dadas por las diferencias de clases sociales, inclusive de tipo religioso, pero el factor político siempre está presente" (Garay y Perez, 2018, p. 90); aunado a ello, se encuentra el impacto generado por diversos eventos generadores de cambio, en tanto "hay modificaciones estructurales o acontecimientos históricos que le imprimen nuevos rumbos a las luchas sociales y políticas"(Sanchez, 1990, p. 12).

El análisis del conflicto armado, en relación con el efecto en las victimas y en las poblaciones donde éste se ha desarrollado, ha permitido conocer las grandes secuelas ocasionadas por este a los habitantes, desde el ámbito físico, psicológico, familiar y social; por ende, también se conoce el atraso económico, las condiciones de temor, la falta de protección, la falta de oportunidades y el riesgo de ser víctima que se genera en medio de las confrontaciones. Tras ello, también se originan los desplazamientos, en su mayoría de manera forzada, lo que produce que se abandonen las tierras, en su mayoría en la ruralidad, dejando de laborar el campo y extinguiendo así las bases económicas de estas poblaciones. 
En todo caso, los conflictos armados siempre han propiciado grandes pérdidas, dimensionando el grado de violencia con el que se desarrollaron; tal como lo aduce Girard (1972), en la violencia y lo sagrado, "cuando no es satisfecha, la violencia sigue almacenándose hasta el momento en que se desborda y se esparce por los alrededores con los efectos más desastrosos" (p. 20); teniendo en cuenta la anterior, se entiende que no ha habido población, que siendo afectada por este flagelo, haya tenido resultados diferentes; solo hasta la culminación, a través de diálogos o acuerdos, se puede dar un nuevo comienzo, el cual indica partir de cero y para ello se requiere de una gran labor para la reconstrucción.

Colombia no ha sido un país ajeno al conflicto armado, originando como respuesta una ola de violencia generada en el enfrentamiento de los simpatizantes de los grupos políticos y como voz de revolución ante las estrategias políticas del gobierno; los efectos de dicho conflicto han sido funestos, al punto de ser catalogado el país dentro de las poblaciones con más alto nivel de desplazamiento forzado, "al lado de afganos, congoleños, iraquíes, nigerianos, somalíes, sudaneses, sursudaneses y yemeníes, con más de 2 millones de personas desplazadas a finales de 2015, ya sea en el interior de sus países o como refugiados o solicitantes de asilo" (ACNUR 2015).

A su vez, en el territorio colombiano el conflicto armado ha presentado fluctuaciones, originadas por el nacimiento de nuevos actores, en un comienzo entre el estado y los grupos guerrilleros; posteriormente, las autodefensas surgieron en las zonas de mayor presencia de la guerrilla, aumentando los enfrentamientos y los actos directos contra la población; además, se suman las organizaciones criminales, las cuales ven en las zonas de conflicto un capital delictivo, por ser estas, zonas propicias para el cultivo, para la elaboración y el tráfico de estupefacientes, para la alta riqueza minera, entre otros motivos propagadores de los actos de violencia, generados en busca del control del territorio.

Por su parte, la zona del Magdalena Medio presentó una fuerte injerencia de los grupos del ELN, EPL y las FARC, produciéndose allí enfrentamientos entre dichos grupos, por el control del territorio; también hubo allí presencia de las autodefensa y del ejercito: "Durante dos décadas, las guerrillas fueron el actor con mayor presencia en la zona, sumado a la llegada del Frente 12 del EPL y del Frente 46 de las Farc" (Verdad Abierta, 2016, párr. 13). El surgimiento de las autodefensas estuvo relacionado con el objetivo de contrarrestar y limitar todo tipo de acciones ejecutadas por las guerrillas; a la postre, se desarrollaron las mismas acciones, intensificando los niveles de violencia, evidenciada esta, por ejemplo, en la masacre de funcionarios, de contrincantes políticos y de campesinos, este último fenómeno, presentado en las marchas de mayo de 1988 (Verdad Abierta, julio 2016, párr. 15).

La violencia a su vez ha sido generadora de pobreza, de terror y de zozobra en toda la región, lo cual se refleja en el desplazamiento masivo, dado con diferentes características, amenazas, abusos e inseguridad; aplicando en este ámbito "La violencia el espacio conflictual es definido(...) en términos de persecución y de diáspora, de huida en múltiples direcciones, del campo a la ciudad, del poblado a la metrópoli (...) de Colombia a las naciones vecinas" (Sanchez, 1990 p. 14). Fue así como el campesino se vio obligado a abandonar sus tierras, fincas y animales y a encaminarse hacia nuevas tierras, sin contar con recursos, ni con medios para el sustento económico.

Enfrentados los diferentes actores, buscando demostrar su poderío y como mecanismo de defensa para impedir el paso del enemigo, se emplearon las minas antipersonales, siendo el ELN, el grupo al que se le ha adjudicado ser el primero en usar estos mecanismos (Verdad Abierta, febrero 2016, párr. 4). La instalación de estas minas, de forma indiscriminada, permitía impedir el acceso a las zonas, además de ocasionar daño a las tropas enemigas; al mismo tiempo, su utilización ocasionó grandes víctimas entre la población civil, con un sinnúmero de afectaciones de tipo personal y social, convirtiéndose este en el mayor motivo de desplazamiento y de abandono de las tierras, por parte de los habitantes de la localidad donde se encontraban dichos artefactos. 
En el reconocimiento de las zonas afectadas por eventos de minas antipersonales se caracterizan en esencia por la ruralidad, con dificultad en las vías de acceso, un alto porcentaje de necesidades básicas insatisfechas, y baja densidad poblacional (Descontamina Colombia, estadísticas). Dichos artefactos, ubicados especialmente en los pasos obligatorios, hacen de su efecto una constante amenaza y, por ello, es necesaria la destrucción, la prohibición de su uso y de su fabricación y comercialización, lo cual se considera una tarea por demás de alta complejidad, riesgo y costo.

\section{Minas antipersonales (MAP)}

La minas antipersonales han sido construidas de forma industrial a nivel mundial, pero, como característica especial en el territorio nacional, los diferentes grupos al margen de la ley y los actores del conflicto armado, se han dado a la tarea de construirlas de forma artesanal (AICMA, 2020);por tal motivo, se hizo más difícil su ubicación, ya que en la medida del menor o mayor uso de metales, se hace posible o no su detección, por parte de los equipos destinados para ello; lo anterior, ha ralentizado el proceso de desminado operacional o humanitario. De acuerdo con Humanitarian Practice Network, elementos presentes en el afianzamiento y en el crecimiento de los grupos armados actores del conflicto, mantienen una relación directa con la vinculación de otras actividades ilícitas, como el narcotráfico, facilitando, desde el orden económico, el sostenimiento, la adquisición de armamento y de elementos para la elaboración de las MAP de tipo artesanal e industrial. El objetivo de quienes han utilizado estos artefactos ha sido resguardar las zonas vinculadas con el cultivo, procesamiento y rutas de distribución de narcóticos (Arango, 2010. parr. 2).

En el artículo $2^{\circ}$ del Tratado de Ottawa, numeral 1 , se describe el alcance del concepto mina antipersona:

Se entiende toda mina concebida para que explosione por la presencia, la proximidad o el contacto de una persona, y que incapacite, hiera o mate a una o más personas. Las minas diseñadas para detonar por la presencia, la proximidad o el contacto de un vehículo, y no de una persona, que estén provistas de un dispositivo antimanipulación, no son consideradas minas antipersonal. (Organización de las Naciones Unidas, 1997, p. 60).

La gravedad de los efectos de las MAP constituye uno de los grandes motivos del desplazamiento forzado, presentado en el conflicto armado, así como en el principal temor a la hora de pensar en volver a sus tierras, luego de las negociaciones de un acuerdo de paz y de los resultados en los procesos de restitución de tierras. Ello, en tanto dichas minas han sido "Diseñadas para mutilar en lugar de matar a un soldado enemigo [...]" (International Campaign to Ban Landmines, s. f. b).

Las estadísticas respecto de este fenómeno en el territorio colombiano no son alentadoras, con un proceso de recopilación de información inexistente, que había impedido la atención o, al menos, el conocimiento de los eventos y sus consecuencias. Por lo tanto, la oficina de Atención Integral Contra Minas (AICMA) se ha encargado de consolidar la base de datos, con el registro de los eventos y de las víctimas por las minas antipersonal; con fecha de corte 31 de octubre 2020:

Se han presentado 11.947 víctimas por minas antipersonal y munición sin explosionar, siendo 2006 el año más crítico, pues se presentaron 1228 víctimas, el mayor número en toda la historia de Colombia. (...) Esta problemática ha dejado heridas al $80,5 \%$ (9626) de las víctimas y el $19.5 \%$ (2321) personas han fallecido a causa del accidente, es decir, 1 de cada 5 víctimas muere. Por otra parte, Colombia ha sido uno de los países del mundo con mayor cantidad de víctimas de la fuerza pública y esto ha significado que del total de víctimas, el $60 \%$ han sido miembros de la fuerza pública y el 40 \% restante, corresponde a civiles (Asistencia Integral a las Víctimas de MAP y MUSE, 2020, parr. 4)

En lo corrido del año 2020 se han presentado 128 víctimas (AICMA Colombia 2020), en eventos presentados de 
acuerdo con varias circunstancias. En primer lugar, en los enfrentamientos generados como causa de la existencia aun del conflicto armado; en otros casos, por las MAP y Municiones Sin Explotar (MUSE) abandonadas, las cuales han sido activadas de forma accidental, al paso desapercibido de los habitantes.

Dadas las características propias de las MAP, por las cuales estas logran sobrepasar los controles, facilitan su transporte y, en especial, logran ser imperceptibles, la gravedad de las lesiones ocasionadas, desde mutilaciones hasta la muerte, afectan no solo a la víctima directa, sino a todo el conglomerado familiar y social. Por lo anterior, a estas se las define de la siguiente manera:

El 'soldado perfecto', pues nunca duerme y nunca falla, no dejan de actuar frente a un cese de actividades bélicas y aunque han sido creadas para fines de guerra, no distinguen entre combatientes, adultos ni niños, pues se observa que sólo el diez por ciento de sus víctimas son combatientes. (Corte Constitucional de Colombia, C- 991-2000, 2.2.1.1. (a) parr. 5).

En el país, los militares están en primer lugar dentro de las víctimas de las MAP, lo cual genera altos costos en la atención y una fuerte baja en las unidades operativas de la institución. Asimismo, según Garay y Pérez (2018 p. 85), de forma independiente a la problemática del daño por MAP, se suman los efectos causados por el desplazamiento, por el reclutamiento y por el aislamiento de poblaciones, como detonantes de la criticidad de la violación de los derechos humanos (DD. HH.), y del derecho internacional humanitario (DIH).

Del mismo modo, las zonas de afectación presentan detrimento en la calidad de vida, a tal punto de originar desplazamientos a otros territorios, como método de supervivencia; es así como "las masacres, asesinatos, secuestros y detenciones", generan un ambiente de abandono, desprotección e incapacidad, al punto de forzar a la población a "abandonar sus tierras y su historia" (Arango, 2010. parr. 6).

El análisis de los derechos afectados por el uso de las MAP incluye también la imposibilidad de acceso a la educación; al respecto, la Corte Constitucional ha señalado lo siguiente:

El derecho a la educación es un derecho fundamental, por cuanto los derechos de los niños y las niñas prevalecen sobre los derechos de los demás y la educación colabora con el libre desarrollo de la personalidad (Constitución Nacional, 1991, art. 44)

Además, se dimensiona la participación productiva y el desenvolvimiento en la sociedad; en tal sentido, "por la situación de debilidad de los niños y las niñas, obliga a su atención especial por parte del Estado" (Romero 2013. pág. 58).

Ejercer el derecho a la educación no existe solo; su desarrollo se da en la implícita relación con otros derechos y, como integrante de la sociedad, implica el "derecho de participación en las decisiones de la comunidad educativa, derecho de defensa y derecho a la integridad personal; esto último significa que no puede ser castigado de manera degradante, cruel o humillante" (Romero 2013, p. 58).

Por otra parte, respecto de la protección del medio ambiente, es importante dimensionar los daños y las consecuencias del uso de las minas antipersonal. Si bien es cierto que dichos artefactos no están dirigidos a la contaminación ambiental, se observa cómo, por causa de su uso, el desplazamiento y el abandono del territorio ha generado una reducción a gran escala de la productividad de la tierra (Organización de las Naciones Unidas, 2005). Por su parte, en 
relación directa con la contaminación ocasionada, esta se da por efecto de filtración a las fuentes hídricas, cuyo consumo se constituye en un fuerte riesgo para la vida animal y humana. A su vez, la erosión ocasionada por la destrucción de la cobertura vegetal y la pérdida de productividad del suelo, por la presencia de nitratos y de nitrógeno amoniacal, en valores superiores a los permitidos para la actividad agrícola, dejan consecuencias negativas (Vega, 2017, p. 9), además del daño morfológico y de composición del suelo y la destrucción de flora y fauna en mayor o menor medida, dependiendo de la clase y de la proporción de explosivos empleados (Gonzales et al. 2020, p. 5). Lo anterior, ha generado graves afectaciones en los niveles de equilibrio físico- químico de todo el ecosistema y, con ello, también incidencias socio económicas y culturales en la comunidad.

\section{Tratado de Ottawa}

El Tratado de Ottawa invita a todos los países a participar en la lucha en contra del uso de las MAP, en el conflicto armado, por cualquiera de sus actores, iniciando por la destrucción de los inventarios dispuestos a nivel estatal; prosigue con la ubicación de las zonas de riesgo o amenaza, donde se han presentado los eventos y las victimas por MAP. Lo anterior, ha exigido la recopilación de los datos de identificación de las víctimas y la inclusión para el reconocimiento y para el resarcimiento de los derechos a la vida, a las libertades y a la seguridad personal, los cuales han sido vulnerados, dando el sentido humanitario a la convención, y estableciendo una especial trascendencia en su implementación. En la historia, la acción humanitaria más conocida corresponde a "Henri Dunant, quien, en 1959, al ver las precarias condiciones de los soldados heridos durante la batalla de Solferino, canceló sus actividades y comenzó a socorrerlos" (Cardona, 2016. p. 4).

A su vez, identificar el excesivo daño ocasionado por las MAP en los conflictos internos como en las guerras, cuyas dimensiones culminan en una afectación degradante y por mucho, en contra de todos los preceptos del Derecho Internacional Humanitario, ha permitido la incorporación de diferentes naciones, en la lucha contra su uso, en la Convención de Ottawa, concretando acciones específicas para su destrucción y erradicación.

Las tareas asumidas en el tratado son muy complejas; abordar el proceso implica generar lo mecanismos de comunicación acertados para la ubicación, conteo, destrucción y reconocimiento, ante lo cual se presenta choque, en primera instancia, por el miedo, además, de las problemáticas generadas por el desconocimiento del proceso, haciendo dispendioso cubrir la totalidad del territorio nacional. En tal sentido, se hizo necesario solicitar la ampliación del plazo y, el pasado 20 de noviembre, este fue aprobado por la Convención de Ottawa, por 4 años y 10 meses, de tal forma que irá del 1 de marzo de 2021 al 31 de diciembre de 2025 (AICMA 2020); ello, para poder cubrir las zonas aun faltantes por el proceso de desminado y la atención integral a las víctimas.

Ahora bien, el análisis de las víctimas de MAP no puede dejar sin valor o respaldo a ninguna de ellas; sin embargo, es necesario contemplar las condiciones especiales presentadas en los niños, las niñas y los adolescentes víctimas, específicamente en zonas que aún están presentando el conflicto armado, o por los artefactos abandonados después del conflicto. Ello, en tanto de "1990 a abril de 2014, se han registrado 10.721 víctimas de estas armas, de los cuales 235 son niñas y 828 niños, es decir, el 10 por ciento del total nacional" (Ayure, Ortíz y Serrato, 2014, p. 4), en razón a la vulnerabilidad de este grupo poblacional, el programa ha desplegado una atención especial, incluyendo la educación para la prevención de los accidentes por manipulación accidental.

En ese mismo orden de ideas, los derechos de todo individuo permiten su libre desarrollo; por ello, su existencia y protección debe darse sin discusión alguna. Sin embargo, en los menores de edad, por su condición de "ciudadanos en desarrollo", cualquier afectación cobra mayor relevancia e impacto. Para Hierro (2004) (citado por Galvis 2006. p. 28), 
es necesario identificar en los niños, las niñas y los adolescentes, su "capacidad de participar, en la vida de sus comunidades (...)", teniendo en cuenta su propio contexto y necesidades, no por ello, menos importantes.

No obstante, la historia ha mostrado cómo se han realizado acciones que atropellan los derechos de los niños y de las niñas en todos los campos, como, por ejemplo, las grandes afectaciones y degradación ocasionada por cuenta de la carrera por la producción industrial, en los países europeos (Galvis, 2009. p. 598).

A pesar de lo anterior, el espíritu del derecho y del entendimiento del ser humano ha consagrado la atención a las víctimas, en todo tipo de conducta delictiva, con una consideración especial, para evitar la revictimización; en consideración con ello, la asistencia de una víctima en la infancia o en la adolescencia, está orientada, en primera instancia, a no generar un efecto negativo, ni en el individuo, ni al conglomerado, para lo cual, se reclama el "conocimiento profundo de la heterogeneidad y complejidad (...)", en todos los aspectos que implican las relaciones de todo individuo (Ayure, et al, 2014. p. 16).

\section{Desminado humanitario}

El desminado humanitario, como la actividad específica de búsqueda, ubicación, detonación y destrucción de las MAP, corresponde a una tarea de saneamiento del territorio, como primer elemento garante del uso de la tierra, sin que los pobladores tengan temor a ser víctimas de los funestos efectos de dichos artefactos. Su desarrollo va acompañado de tareas educativas para minimizar los riesgos y de programas para las labores del campo, comprendiendo también la comercialización de los productos.

El proceso busca generar las condiciones de recuperación de la movilidad, uso y transporte, en la reconstrucción social y económica de la comunidad, actividad regulada por la Instancia Interinstitucional de Desminado Humanitario, creada mediante el Decreto 3750 de 2011, "su objetivo es eliminar los peligros derivados de las minas antipersona (MAP) [...] con el fin de restituir las tierras a la comunidad para su utilización" (Glosario Nacional Básico General de Términos de Acción Integral contra Minas Antipersonal, 2012, p. 5).

Una de las complicaciones del proceso de desintoxicar el territorio colombiano de artefactos explosivos, es la diversidad de los tipos de minas artesanales, las cuales son construidas con mínimas cantidades de material metálico, lo que hace difícil su detección y su consecuente extracción; además, los costos del desminado son elevados, por lo tanto, "fabricar una mina cuesta un dólar, sin embargo, hallarlas y desactivarlas cuesta hasta mil veces más". Lo anterior, sin contar el riesgo permanente que tienen los desminadores; "Por lo menos 7000 han caído en ellas en los últimos 15 años, de los cuales 1400 han muerto" (Ruiz, 2015, p. 34).

Por su parte, existe el desminado operacional, el cual es llevado a cabo para permitir el desarrollo de actividades, misiones o desplazamientos, no dentro de la planificación de Descontamina Colombia, pero si como forma de contribuir en la destrucción de las MAP, evitando nuevas víctimas por dichos artefactos; así bien, "el desminado militar puede convertirse en una acción humanitaria que busca destruir la amenaza de los artefactos explosivos, sin que ello signifique una ventaja militar" (Cardona 2016, p. 12).

A su vez, los planes de acción del desminado humanitario, trazados con la suscripción a la convención de Ottawa, han implicado muchos retos y la vinculación de diferentes organizaciones internacionales, con conocimiento en el proceso de desminado, obedeciendo a los parámetros establecidos por el IMAS, basándose en los principios propuestos en Dinamarca y en los Estándares Internacionales para Operaciones Humanitarias de Despeje de Minas, creados a finales 
de 1996 (Cardona 2016, p. 6). Lo anterior, incluyendo metodología específica, monitoreo y control, para cubrir todo el territorio nacional y poder declararlo libre de sospecha de minas.

En tal sentido, el desarrollo de los planes de desminado humanitario tiene como fin liberar todo espacio con sospecha de minas antipersonales y, con ello, asegurar "la movilidad de la comunidad en riesgo y [permitir] la restauración de derechos de las comunidades" (Orjuela, 2015, p. 25); no obstante, dicha actividad en sí misma y sin la aplicación de otras medidas no constituye en sí la solución de los problemas, puesto que "el desminado es complementario con otras políticas públicas, como es el caso de las políticas de restitución de tierras y retornos,(...)". Por lo anterior, el desarrollo del desminado humanitario debe abarcar los diferentes roles que cumplen las personas, en las zonas a intervenir, entre ellas el desminador, la comunidad, el mando militar, el cooperante y la(s) autoridad(es) municipal(es) (Corcione y Macías 2020, p. 162).

El proceso mecánico del desminado realizado por las diferentes entidades del orden nacional o internacional, certificadas para tal fin, no es nada fácil. Este implica la capacitación de personal, la adquisición de medios y de recursos para su aplicación, lo cual además es una tarea lenta y de mucho cuidado, e involucra la exposición y riesgo de manera permanente de las personas que lo desempeñan. No obstante, con el valor que caracteriza al colombiano, los ciudadanos muchas veces se enfrentan a estas condiciones, no con otra intención, que con la de liberar cada centímetro del territorio de este flagelo, para así permitir a las familias recuperar sus espacios, sus propiedades, sus recuerdos, sus formas, así como con el objetivo de mejorar las condiciones de calidad de vida.

De igual modo, se entiende que, siendo referente en el diseño de las estrategias por su carácter olístico e integral, es importante tener en cuenta que las características propias de la geografía colombiana no permiten la aplicación de todos los medios tecnológicos creados para tal fin, haciendo que el desarrollo de las tareas de desminado sea más lenta y aumentando los riesgos para el personal de desminadores.

\section{Postconflicto}

El acuerdo de paz ha generado nuevas condiciones en la percepción de seguridad, al eliminar las condiciones de riesgo, al mostrar mejoras en la calidad de vida, en la recuperación de las tierras y de los territorios, así como en propiciar ambientes de asistencia para el desarrollo productivo y empresarial.

El posconflicto, definido para el contexto colombiano como el período de tiempo posterior al termino del conflicto armado (total o parcial), en el cual se alcanzó la reducción estadística de homicidios generados por el conflicto, "por debajo de un umbral determinado" (Universidad del Rosario, s. f.), no abarca muchas otras condiciones dadas en el entorno; por tanto, para ubicarlo en la realidad actual, es necesario propender por generar condiciones de construcción social de alto compromiso.

Así bien, crear verdaderas condiciones de paz incluye el desminado humanitario, visualizado como un elemento importante en la disminución y en la erradicación de cualquier riesgo, para que a su vez, esa disminución de riesgos permita la restitución de las tierras y, con ello, el impulso de nuevo a las labores en el campo, a las actividades de producción y de sostenimiento económico familiar, así como a la creación de empresas de transporte y de comercio, tanto como al desarrollo de las actividades agrícolas y pesqueras, y en búsqueda de generar espacios de turismo, siendo estos los factores que son favorables para los territorios. En tal sentido, se requiere de políticas claras, las cuales van más allá de la reducción de las estadísticas de homicidios, logrando articular todos los aspectos de reconstrucción y de superación de los índices de necesidades básicas insatisfechas. 
Así mismo, el posconflicto exige condiciones únicas, las cuales deben estar dirigidas a "la acción y rehabilitación de las víctimas, incluir la cooperación internacional para agilizar y garantizar el avance concluyendo en la asistencia humanitaria (Garzón, Parra \& Pineda, 2003, p. 21). En este sentido, la estrategia colombiana ha sido un referente, en tanto abarca la atención a las víctimas en todos los ángulos posibles, especialmente en la capacitación y la educación, en primera instancia, para prevenir víctimas. Lo anterior, además estableciendo procedimientos y el reconocimiento de las minas antipersonal, especialmente entre los niños, quienes se han visto sumamente afectados. Ello implica la atención a las víctimas de forma integral, lo cual es una tarea compleja, contemplando las graves secuelas y las grandes limitaciones generadas a las víctimas.

En esa misma línea, formular espacios ciertos para el posconflicto y poder generar en el ciudadano la credibilidad en un proceso de paz, requiere de las condiciones de seguridad, presencia del Estado, estrategias y prácticas de crecimiento, apoyo oportuno e integración con el campesino, así como el mejoramiento de vías, aportes económicos, asesoramiento para el trabajo en el campo; además del impulso a la producción y la comercialización de los productos, con estándares de calidad válidos para la exportación.

La elección de los mecanismos de reconstrucción y de rehabilitación resulta esencial, para poder establecer condiciones reales de participación, en un esquema posbélico, basado en los aspectos social, político e institucional, con dirección al orden humanitario (Garzón, et al., 2003, citado porArias et al. 2018 p. 22).

Lo anterior, mediante estrategias adecuadas para la concertación y la negociación, con la "participación de los distintos actores armados y sociales que hacen parte del conflicto" (Pérez, 2011, p. 135), dimensionando una gran estela de posibilidades de recuperación.

Las actividades de incorporación y participación social deben darse de forma permanente, en la consolidación de la reconstrucción social como el proceso de afianzamiento de la paz, sin dar opciones a volver a las condiciones de violencia que se presentaban durante el conflicto (Rettberg, 2003 p. 16).

Se genera el desarrollo económico con nuevas formas de comercio y, en especial, de los productos propios de cada región y lo social desde la participación en la implementación de estrategias dirigida a la satisfacción de las necesidades manifiestas. Rettberg (2003 p. 17).

La declaratoria de un territorio "libre de minas" tiene tal trascendencia, no solo respecto de la acción física de retirar todos los artefactos de la localidad, sino también en relación con el ensamble, siguiendo un camino de reconstrucción social y de reconocimiento a las víctimas, en la afectación de sus derechos, para la recuperación de sus propiedades, y para que estos puedan volver a casa, en condiciones de tranquilidad y de seguridad. Es decir, con la intención de transformar las vivencias de violencia en una nueva ruta de convivencia familiar y económica, así como con el objetivo de lograr el reencuentro y la satisfacción de las necesidades básicas y esenciales de las víctimas.

\section{Unidad de restitución de tierras: URT}

La restitución de tierras en Colombia es una acción de la política de atención a las víctimas del desplazamiento forzado, cuya esencia es devolver la tierra a los campesinos y a las comunidades étnicas, quienes fueron obligados ha abandonarlas.

Esta se sustenta en La ley 387 de 1997, con la aplicación de medidas para la prevención y para la atención del 
desplazamiento forzado. Posteriormente apareció la Ley 1448 de 2011, que versa sobre la "atención, asistencia y reparación integral a las víctimas del conflicto armado interno", la cual surge como respuesta a las solicitudes de la Corte Constitucional, institución que, en el auto de seguimiento No. 008 de 2009, exhortó al Estado colombiano a implementar mecanismos para conocer y resolver las reclamaciones de restitución de tierras de la población desplazada.

En Colombia se cuenta, desde 1980, con la participación de la Agencia de Cooperación Internacional de Japón JICA, cuya prioridad es el fortalecimiento del capital humano, a través de la transferencia de tecnología y conocimiento, como política dirigidas a la atención de temas de relevancia; estas acciones son concertadas con el Gobierno colombiano, para que su impacto sea aplicable y sostenible en el tiempo (Unidad de Restitución de Tierras, 2020, p. 13).

Una vez se materializa la restitución de las tierras se promueve la sostenibilidad socioeconómica, a través de la implementación de sistemas de producción que sean acordes con las características agro-climatológicas, con la logística y con las actividades comerciales de cada región.

Lo anterior, logrando la reactivación productiva de 4.354 familias y de 34.541 hectáreas, en 171 municipios de 21 departamentos, con la producción de alimentos como el aguacate, el cacao, el café, la caña, el ñame, el tomate, el limón y el plátano, así como con ganadería de levante, ganadería de leche, ganadería de ceba, ganadería de doble propósito, piscicultura, porcicultura y avicultura (Unidad de Restitución de Tierras, 2020. p. 82)

\section{San Vicente de Chucurí}

San Vicente de Chucuri es conocido a nivel nacional por sus productos agrícolas, en especial por el cacao y el aguacate, cuyas características los distinguen de las demás clasificaciones habitualmente consumidas en el territorio nacional.

Dicha región fue constituida como Municipio por medio de la ordenanza Departamental No. 16 del 8 de agosto de 1890, de la Asamblea de Santander. Su fundación se establece con fecha del 7 de septiembre de 1876, la cual es tomada a partir de registros históricos, según el acuerdo 014 de 29 de agosto de 2005 (Plan de Desarrollo 2.016-2.019. p. 32)

Esta región cuenta con una población total de "treinta y cuatro mil ciento dieciséis (34.116)" personas, y con un total de personas consideradas víctimas de la violencia, de dos mil cuatrocientas cuarenta y nueve (2.449) personas (Plan de Desarrollo 2.020-2.023. p. 30).

La actividad agropecuaria es su principal actividad de ingreso económico, junto con actividades de tipo pecuario y, en una baja proporción, de minería.

Esta región ha sido considerada ampliamente como despensa agrícola del departamento, como tierra de los frutos valiosos y, además, ha sido reconocida como la capital cacaotera del país, contando con un área actual cultivada de 15.696 hectáreas, en la cual se encuentran además varias especies como el aguacate, los cítricos y el plátano (Plan de Desarrollo Municipal 2020-2023, p. 39).

Por su parte, San Vicente de Chucuri sufrió todos los infortunios de la violencia, la cual fue generada por ser este el lugar de nacimiento de la guerrilla; allí se originaron las contiendas entre los simpatizantes de los grupos y los integrantes de los partidos políticos e instituciones, dando lugar a enfrentamientos en los cuales la población civil quedó en medio de dichas disputas, sufriendo graves consecuencias por causa de estos vejámenes. Esta ha sido una marca que ha acompañado por mucho tiempo a sus habitantes, a tal punto de tener estos que trasladarse a lugares aledaños, para 
registrar los nacimientos, con el fin de no ser señalados como guerrilleros (VerdadAbierta.com 2016, párr. 6).

En palabras de Vargas (1989), la historia de la violencia en Colombia "nos enfrentan a un sinónimo de continuidad y rupturas" (p. 34); lo anterior, también en el contexto de la historia de la colonización intrarregional, generada en San Vicente de Chucuri. Lo anterior se dio inclusive hasta presentar los actos violentos en el origen del conflicto armado, con la consolidación del grupo guerrillero rural, el cual, para "el 4 de julio de 1964 (fue) denominado como Ejército de Liberación Nacional del municipio de San Vicente de Chucuri” (Vargas, 1989. p. 35).

Por su parte, en dicha región el empleo de las MAP afectó a gran parte de la población rural, la cual fue la más afectada, dada la facilidad para ocultar las MAP en ese tipo de lugares. Lo anterior produjo altos niveles de desplazamiento entre la comunidad en general, e implicó una dificultad para que las autoridades y los servicios médicos pudieran tener acceso a aquella, y para lograr su cubrimiento y atención, lo que a su vez produjo un alto índice de victimas. En tal sentido, el municipio lo constituyen "37 veredas de las cuales 14 estaban plagadas de minas\{...\}"; la ubicación de estas se encontraba en sectores estratégicos, tales como vías de acceso y pasos obligatorios, con el fin de lograr un mayor impacto y mejores resultados bélicos(Verdad Abierta, febrero 2016, párr. 15).

Siguiendo esta línea, allí, la actividad del desminado logró la remoción de noventa (90) minas antipersonal y de otros diez (10) artefactos explosivos. De acuerdo con los datos de AICMA, la estadística de Acción Contra Minas 2020, en San Vicente de Chucuri, reporta la información que se puede leer a continuación en la tabla 1.

\section{Tabla 1.}

Estadística de víctimas por minas antipersonal.

\begin{tabular}{lc}
\hline Tipo de evento & Número de víctimas \\
\hline Víctimas civiles & 16 \\
Víctimas de la fuerza pública & 11 \\
Víctimas femeninas & 0 \\
Víctimas másculinas & 24 \\
Víctimas sin información de genero & 3 \\
Víctimas heridas & 23 \\
Víctimas muertas & 4 \\
Víctimas mayores de 18 años & 25 \\
Víctimas menores de 18 años & 2 \\
Víctimas totales & 27 \\
\hline
\end{tabular}

Fuente: Estadística Acción Contra Minas (2020)

\section{Análisis de desarrollo del municipio. Índice de competitividad municipal 2018-2019.}

La medición de competitividad permite confrontar las condiciones de cada municipio, en relación con la satisfacción de las necesidades básicas, teniendo en cuenta aspectos como la educación, la infraestructura, la salud, entre otros. Ello, con la finalidad de identificar necesidades o falencias y de demarcar un derrotero a seguir, en la consecución del mejoramiento de la localidad.

En la medición realizada por el Consejo Privado de Competitividad para 2018-2019, se ubicó en el tercer lugar al departamento de Santander. Con la participación de las Cámaras de Comercio de Bucaramanga y de 
Barrancabermeja, Fenalco Sur de Santander, Crezcamos, la Universidad Libre, Unisangil y la Comisión Regional de Competitividad de Santander, se realizó el análisis de los municipios del departamento. Allí se estipularon las valoraciones recibidas para los 30 primeros municipios; no obstante, entre ellos no aparece el municipio de San Vicente de Chucurí, a diferencia de Barrancabermeja y del Carmen de Chucurí, regiones que son próximas a dicho territorio.

Por otra parte, en el municipio de San Vicente de Chucuri se desarrollan programas de caracterización y de atención a las víctimas del conflicto armado, del desplazamiento y a la población vulnerable; con ello se logra la organización de diferentes asociaciones, de acuerdo con las actividades o proyectos a realizar por su ubicación, y que también estén dirigidas a desarrollar actividades de recopilación de datos, como lo hace la fundación Circulo de Estudios Culturales y Políticas. Este mecanismo permite obtener capacitación, asistencia y recursos.

Así mismo, el macroproyecto de la Hidroeléctrica Sogamoso ha permitido el crecimiento del turismo, posicionándolo como un componente económico fuerte del municipio; ello trae consigo la creación de empresas, nuevas formas para la comercialización de los productos propios de la región y, por ende, otros medios de ingreso económico.

\section{Proyectos productivos}

El éxito en los proyectos productivos se ve afectado por diferentes variables, como lo son el desconocimiento en general de su implementación, el abandono al cual está sometido el sector agrícola, la falta de recursos para el desarrollo del proceso y, en especial, no poder concretar la etapa de comercialización de los productos, siendo esta una de las grandes dificultades. A ello se suman las condiciones de vías de acceso y los medios de transporte, desde las zonas rurales hacia los mercados.

Por su parte, la reconstrucción social requiere de políticas de implementación de proyectos productivos, como soporte y asistencia a la comunidad, permitiendo la organización y un manejo adecuado de los recursos, llevando el proceso hasta el aseguramiento de la comercialización de los productos.

\section{Análisis delictivo}

El análisis de los delitos de mayor impacto del municipio de San Vicente de Chucuri2014-2019, comprendiendo la estadística del antes, el durante y el después del proceso del desminado humanitario se emplea como recurso para observar el comportamiento de las condiciones de seguridad de la población.

Desde la ausencia del conflicto armado no se presentan casos de atentados, pero sí se observa el aumento de delitos de impacto como el homicidio, para los años 2018 y 2019, con dos casos cada año.

Sobre el delito de extorsión este se presenta con un caso para el año 2014; para el año 2016 dos casos; para el 2018 cuatro casos y para el año 2019 dos casos.

Para los delitos de impacto en la seguridad ciudadana se observa un incremento en el año 2018 y para el 2019 vuelve a presentarse una reducción, quedando con cifras por debajo de los presentados en el año 2014, con mayor incidencia en los casos de lesiones personales, hurtos a vehículos y hurto a personas.

En los casos que afectan la seguridad vial se presenta un alza en el año 2017, con 24 casos, con una notable disminución de más del 50\% para el año 2019, con 11 casos, quedando por debajo del año 2014, el cual había presentado 31 casos, teniéndose un mayor índice en los casos de lesiones en accidentes de tránsito. 
Del total de casos presentados entre el 2014 al 2019, el año 2018 presenta el mayor número de accidentes, con 213 casos, seguido por el año 2017 con 195 casos.

El aumento de la población con el regreso de quienes sufrieron el desplazamiento y la llegada de quienes ven la localidad como una posibilidad de crecimiento, trae consigo nuevas formas de generación de empresa, de organización y de comercio, entre otros aspectos; no obstante, lo anterior implica nuevas condiciones de riesgos, los cuales deben ser analizados de manera continua, para determinar las políticas de acción acertadas en su prevención y atención.

\section{CONCLUSIONES}

El compromiso asumido por Colombia ante el acuerdo de Ottawa implica la participación de todos los actores, en el desarrollo de las tareas para la atención integral de las víctimas y para consolidar condiciones para el incremento de la seguridad y de la convivencia.

El proceso del desminado humanitario representa una de las actividades fundamentales para garantizar el ejercicio de los derechos y de las necesidades básicas de todos los individuos, atendiendo a resolver insuficiencias relacionadas, por ejemplo, con la libertad de movimiento, con la locomoción, con la educación, con la vivienda y con la salud.

El municipio de San Vicente de Chucurí presenta condiciones para su desarrollo, por lo cual las acciones deben dirigirse hacia la potencialización de las actividades productivas de la localidad, contando con la asistencia técnica, con la intervención de las vías de acceso y con los medios de transporte, lo cual pueda permitir la comercialización de los productos, como medio de ingreso y de sustento económico.

El nuevo plazo para la cobertura nacional, con el fin de lograr el proceso de desminado humanitario, permite avanzar en la atención integral a las víctimas; también en dinámicas como la educación para la prevención, en la restitución de tierras y en los proyectos productivos, como mecanismos generadores de construcción social, a través de los cuales, en alineación con las políticas locales y regionales, se pueden obtener verdaderos resultados de éxito.

Finalmente, el proceso del desminado humanitario tiene impacto directo en la reducción de los índices delictivos de la localidad; también contribuye al mejoramiento de la percepción de seguridad, así como al desarrollo de las actividades diarias en el ámbito social, económico y de convivencia.

\section{REFERENCIAS BIBLIOGRÁFICAS}

Acción Contra Minas. (2014) Guía para la Asistencia Integral de los Niños, Niñas y Adolescentes Víctimas de Minas Antipersonal (MAP), Municiones sin Explotar (MUSE) y Artefactos Explosivos Improvisados (AEI). Programa de Acción Integral contra las Minas Antipersonal. www.accioncontraminas.gov.co

ACNUR. (2015). Tendencias globales del desplazamiento forzado en 2015. ACNUR. https://www.acnur.org/fileadmin/Documentos/Publicaciones/2016/10627.pdf

Alcaldía de San Vicente de Chucurí. (2016). Plan de Desarrollo San Vicente somos Todos 2016-2019. Alcaldía de San Vicente de Chucurí. https://sanvicentedechucurisantander.micolombiadigital.gov.co/sites/sanvicentedechucurisantander/content/files/ 000004/188_pdm_20162019_mod.pdf 
Alcaldía de San Vicente de Chucurí. (2020). Plan de desarrollo San Vicente avanza con equidad 20202023.Alcaldía de San Vicente de Chucurí.

https://sanvicentedechucurisantander.micolombiadigital.gov.co/sites/sanvicentedechucurisantander/content/files/ 000716/35784_pdm-san-vicente-avanza-con-equidad.pdf

Arango, A. (2010) La crisis de las minas antipersonal en Colombia. Revista de Intercambio Humanitario. Humanitarian Practice Network. https://odihpn.org/magazine/la-crisis-de-las-minas-antipersonal-en-colombia/

Arias, D. P. \& Ospina Perdomo, J. M. (2020). Desminado humanitario en los escenarios coyunturales del posconflicto colombiano: una mirada jurídico-política. Desafíos Revista Universidad del Rosario. 32(1). http://dx.doi.org/10.12804/revistas.urosario.edu.co/desafios/a.6389

Armiño, J. A. (2006). Acción humanitaria: concepto y evolución. Universidad del País VASCO. http://www.dicc.hegoa.ehu.es/listar/mostrar/1\#

Ayure, Serrato y Ortíz. (2014). Guía para la atención integral de los niños, niñas y adolescentes víctimas de minas antipersonal (MAP), municiones sin explotar (MUSE) y artefactos explosivos improvisados (AEI). Acción Contra Minas. Colombia www.accioncontraminas.gov.co

Cardona, M. (2016), Diseño de un plan de reconocimiento de desminado operacional bajo normatividad IMAS. [Tesis de especialización, Universidad Militar Nueva Granada]. Repositorio Universidad Militar Nueva Granada. https://repository.unimilitar.edu.co/handle/10654/21089.

Cabrera, A. y Pachón, W. (2017). Impacto socioeconómico del desminado humanitario. Análisis de los casos de restitución de tierras de las comunidades de San Francisco y San Carlos (Antioquia, Colombia). Universidad Militar Nueva Granada. TraHs Números especiales N¹. Conflictos y procesos de paz: el caso de Colombia. P. 75-85. http://www.unilim.fr/trahs - ISSN: 2557-0633.

Cámaras de Comercio de Bucaramanga y Barrancabermeja, Comite Regional de Competitividad, Universidad de San Gil, Fenalco sur de Santander, Crezcamos \& Universidad Libre. (2019). Índice de Competitividad Municipal Santander 2018-2019. Categoría: competitividad Santander. www.santandercompetitivo.org/biblioteca-dedocumentos/competitividad-en-santander/ndice-de-competitividad-municipal-para-santander-2019pdf.

Comité Internacional de la Cruz Roja. (2012) Minas antipersonal. Comité Internacional de la Cruz Roja. https://www.icrc.org/es/guerra-yderecho/armas/minas-antipersonal.

Corte Constitucional de Colombia. Sentencia C-991 de 2000. M. P.: Álvaro Tafur. https://www.corteconstitucional.gov.co/relatoria/2000/C-991-00.html

Departamento Administrativo Nacional de Estadística -DANE. (2019). Medida de pobreza multidimensional municipal de fuente censal - Indicadores. DANE. https://dane.maps.arcgis.com/apps/MapJournal/index.html?appid=54595086fdd74b6c9effd2fb8a9500dc.

Dirección de Investigación Criminal e Interpol. Área de Investigación Criminológica. Estadística Delitos de Impacto Municipio de San Vicente de Chucuri 2014-2019.

Frías, A. (1988). Los bolcheviques de 1929. El Yariguí Chucureño, San Vicente de Chucurí, 33-46. 
Galvis, L. (2006). Las niñas, los niños y los adolescentes. Titulares activos de derechos. Ediciones Aurora. https://revistas.unal.edu.co/index.php/anpol/article/view/74239

Galvis, L.(2009). La Convención de los Derechos del Niño veinte años después. Revista Latinoamericana de Ciencias Sociales, Niñez y Juventud, 7(2), 587-619. https://www.redalyc.org/pdf/773/77315614002.pdf

Garay C., y Pérez, A. (2018). Los derechos humanos en Colombia. Revista Científica General José María Córdova, 16(23),83-105. https://doi.org/10.21830/19006586.307.

https://revistacientificaesmic.com/index.php/esmic/article/view/307

Garzón, J., Parra, A. \& Pineda, A. (2003). El posconflicto en Colombia: coordenadas para la paz [Trabajo de grado en Derecho, Pontificia Universidad Javeriana]

Girard, R., (1972). La Violencia y lo sagrado, Colección Argumentos. https://www.anagramaed.es/libro/argumentos/la-violencia-y-lo-sagrado/9788433900708/A_70

GIZ., Alcaldía de San José de Cúcuta., CORPONOR, Cooperación Alemana, ASOJUNTAS. (2018). Mecanismos de concertación social entre actores relevantes para el fomento de la conservación de la zona Sur de Tibú y Norte de Cúcuta 2017. Sociedad Alemana para la Cooperación Internacional, 20.

https://ww2.ufps.edu.co/public/archivos/pdf/4885dbe3a4252e7a7a8fe77feab74e35.pdf

González Forero, R., Romero Guerra, G. D., \& Echeverría Vargas, M. Ángel. (2020). Impacto ambiental en suelos sometidos a explosivos en San Luis, Tolima (Colombia). Logos Ciencia \& Tecnología, 12(3), 47-56. https://doi.org/10.22335/rlct.v12i3.1254

Gutiérrez, S. (3 de abril de 2015). ¡Remángate! en contra de las minas antipersonal en Santander. Radio Nacional de Colombia. http://www.radionacional.co/noticia/remangate-en-contra-de-las-minas-antipersonal-en-santander

Informativo Insignia. (2016). Como se hace el desminado humanitario. [archivo de video]. https://www.youtube.com/watch?v=YP8c5otOalc.

Ley 1448 de 2011. (10 de junio 2011). Congreso de la República. . https://www.unidadvictimas.gov.co/es/ley-1448de 2011/13653\#: : :text=Rese \%C3\%B1a\%3A,y\%20se\%20dictan\%20otras\%20disposiciones.

Macías Montoya, J., \& Corcione Nieto, M. (2020). Experiencias de procesos de desminado humanitario en Colombia desde la perspectiva de los desminadores. Revista Científica General José María Córdova, 18(29), 161-179. http://dx.doi. org/10.21830/19006586.530. Publicado en línea: 1 de enero de 2020.

Marín, I., Prieto, C. y Rocha, C. (2014). Seis Tesis sobre la evolución reciente del conflicto armado en Colombia. (23) Fundación de Ideas para la Paz. https://www.ideaspaz.org/publucations/posts/1053.

Naranjo, G. (2001). El desplazamiento forzado en Colombia, reinvención de la identidad e implicaciones en las culturas locales y nacionales. Revista Electrónica de Geografía y Ciencias sociales, 94(1).

Nuestros Héroes. (2017). Desminado Humanitario. [archivo de video]. https://www.youtube.com/watch?v=6S2QGCB8ax4\&t=30s 
Observatorio de Minas Antipersonal. (2003) Santander. ( $\left.N^{\circ} 3\right)$. Observatorio de Minas Antipersonal. https://repositorio.gestiondelriesgo.gov.co/bitstream/handle/20.500.11762/20457/Del\%20observatorio\%20de\%2 Ominas\%20antipersonas\%20No\%203.

Oficina del Alto Comisionado para la Paz. Descontamina Colombia. Oficina del Alto Comisionado para la Paz. https://www.accióncontraminas.gov.co/Estadísticas/Paginas/Estadisticas-DH.aspx.

Oficina del Alto Comisionado Para la Paz. Tratado de Ottawa. Oficina del Alto Comisionado para la Paz. https://www.accióncontraminas.gov.co/AlCMA/Paginas/convencion-de-Ottawa.aspx.

Oficina del Alto Comisionado Para la Paz. Descontamina Colombia. Oficina del Alto Comisionado para la Paz. https://www.accióncontraminas.gov.co/prensa/Paginas//Alto-Comisionado-Paz-solicita-nueva-prorroga-paracumplir-con-convencion-de-Ottawa-sobre-prohibicion-minas-antipersonal.aspx.

Orjuela, M. (17 de junio de 2015d). Desminado y Ley de Víctimas hacen parte de la entrada del posconflicto: Santos. Radio Nacional de Colombia. http://www.radionacional.co/noticia/desminado-yley-de-victimas-hacenparte-de-la-entrada-del-posconflicto-santos

Pecaut, D. (1997). Presente, pasado y futuro de la violencia. Análisis Político, 30, 3-36. https://revistas.unal.udi.edu.co/index.php/anpol/article/view/76353/68685.

Pécault, D. (1999). La perdida de los derechos, del significado de la experiencia y de la inserción social. A propósito de los desplazados en Colombia. Estudios Políticos, 14, 13-31. https://www.acnur.org/fileadmin/Documentos/Publicaciones/2007/5151.pdf

Palacios, S. y Rodríguez, L. (2015). Paz, posconflicto y sus elementos característicos. Un acercamiento conceptual. Universidad San Buenaventura.

http://bibliotecadigital.usb.edu.co/bitstream/10819/3033/1/Paz_posconflicto_elementos_palacios_2015.pdf.

Rettberg, A. (2003). Diseñar el futuro: una revisión de los dilemas de la construcción de paz para el postconflicto. Revista de Estudios Sociales, 15-28. https://revistas.uniandes.edu.co/doi/pdf/10.7440/res15.2003.01

Romero, F. (2013). Conflicto armado, escuela, derechos humanos y DIH en Colombia. Análisis político, 77, 57-84. https://revistas.unal.edu.co/index.php/anpol/article/view/44001

Sánchez, G., (1990).Guerra y Política en la Sociedad Colombiana. Análisis político, 11, 12. https://revistas.unal.edu.co/index.php/anpol/article/view/74324/67150

Tratado de Ottawa. (1997). Convención sobre la prohibición del empleo almacenamiento, producción y transferencia de minas antipersonales y sobre su destrucción. Santander.

(N³).https://www.accióncontraminas.gov.co/AICMA/Paginas/Convencion-de-Ottawa.aspx.

Ugarriza, J. (2013). La dimensión política del postconflicto: discusiones conceptuales y avances empíricos.

Colombia Internacional, 77, 141-176. https://doi.org/10.7440/colombiaint77.2013.06

http://colombiainternacional. uniandes.edu.co/view.php/9125/index.php?id=9 125. 
Vargas Velásquez, A. (1989). Tres momentos de la violencia política en San Vicente de Chucurí (de los bolcheviques del año 29 a la fundación del ELN). Análisis Político, (8), 33-48. https://revistas.unal.edu.co/index.php/anpol/article/view/74239/67082/393949

Vega, C. (2017). Problemas Ambientales y de Salud derivados del uso de fertilizantes nitrogenados. [Tesis Universidad de Complutense]. Repositorio Universidad de Complutense. http://147.96.70.122/Web/TFG/TFG/Memoria/CRISTINA\%20VEGA\%20OLIVA.pdf

Verdad Abierta. (11 de febrero de 2016). San Vicente de Chucurí y las minas del ELN. Verdad Abierta.,https://verdadabierta.com/san-vicente-de-chucuri-y-las-minas-del-eln/

Verdad Abierta. (12 de julio 2016). El sabor amargo del conflicto en San Vicente de Chucuri. Verdad Abierta. http://El sabor amargo del conflicto en San Vicente de Chucurí | VerdadAbierta.com 